\title{
Control of stenting procedures with optical imaging
}

\author{
Anna Cysewska-Sobusiak* \\ Division of Metrology and Optoelectronics, Institute of Electrical Engineering and Electronics, \\ Poznan University of Technology, Piotrowo 3a, 60-965 Poznan
}

Received April 06, 2020; accepted June 22, 2020; published June 30, 2020

\begin{abstract}
The article presents how application of optical methods of imaging may help control a more and more widespread stenting procedure. Modern gastro-videoendoscopy is especially useful in such therapeutic procedures performed in particular parts of the alimentary tract. Selected examples relate to the results of real studies made in cooperation with medical specialists.
\end{abstract}

Optoelectronic and photonic methods and devices are widely used in biomedical applications. The author has long experience in investigating the utility of light-tissue interaction. Efficient cooperation with a medical staff made it possible to obtain reliable results under clinical conditions. Photonic techniques allowed us to use the optical properties of tissues or organs for their imaging [1, 9]. The main purpose of this paper is to present some selected examples of the effects obtained during real interventions assisted by the optical technique to improve the so-called combined imaging of the stented site.

Stenting is the placement of a stent inside a particular part of the human body. Stents improve the quality of life for patients with various diseases. A stent is inserted into a blocked vessel or another tract to restore the flow of blood or other media. Stents in arteries of the heart have been used for many years. These devices open arteries and allow blood to flow. Now stenting may be also used in the alimentary tract to open blockages caused by a tumor [3, 9, 10]. For example: the self-expandable esophagus stent considered here allows food to flow through freely.

In 1895 Wilhelm Conrad Roentgen made the first radiogram of the palm of the hand, starting the development of noninvasive image diagnostics methods. Currently, different imaging methods are able to detect different properties of investigated tissues through a variety of phenomena utilized. Medical imaging helps physicians to evaluate an area of the human body that is not normally visible. Particular methods differ from the point of view of advantages and disadvantages, range of applications, degree of invasive or noninvasive interaction, patient ballast, and complication of procedures. Thus, effectiveness and precision can be especially improved when some combined methods of imaging are used. As the present tendency shows, the role

\footnotetext{
*E-mail: anna.cysewska@put.poznan.pl
}

of optical techniques in developing combined medical imaging is especially significant, e.g., in advanced videoendoscopy $[3,5,9]$. Optical image resolution can be lower than that of X-ray images; however, it enables to provide information on functional conditions unavailable in the RTG technique. Modern gastro-videoendoscopy is especially useful in minimally invasive diagnostic and therapeutic effects (Fig. 1).
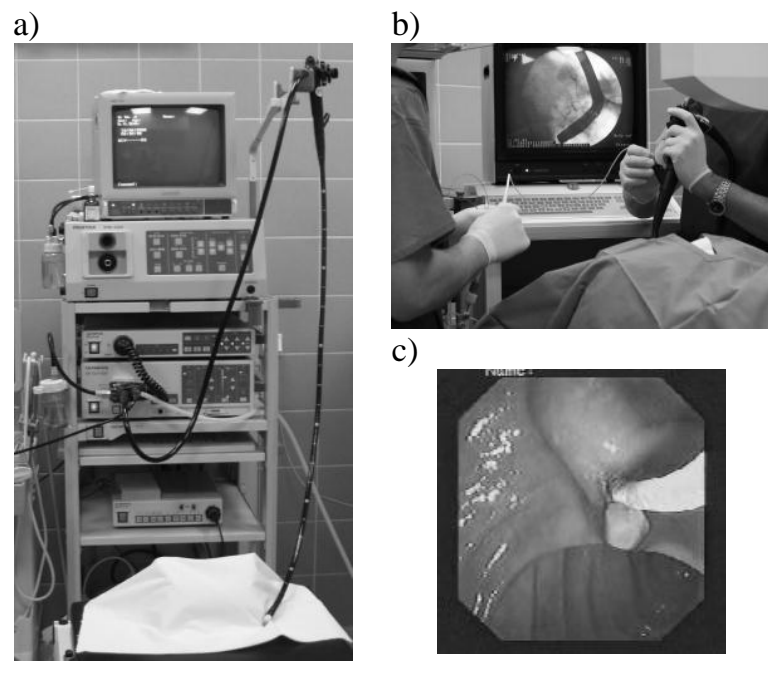

c)

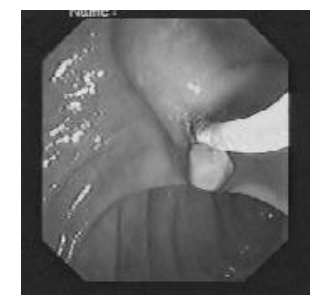

Fig. 1. a) View of a modern gastro-videoendoscope, b) example of minimally invasive fiberoscopy procedures, c) image illustrating a selected step of endoscopic extraction of a gallstone.

Videoendoscopes are computer-assisted devices equipped with a flexible fiber-optic catheter probe tips, at the ends of which there are high-resolution CCD microcameras that allow for direct image transmission from inside the human body. High-performance miniature color CCD video cameras are manufactured for endoscopy in $1.8 \mathrm{~mm}$ and $3 \mathrm{~mm}$ sizes. Offering excellent image quality, the cameras are suitable as visualization devices for companies that wish to develop their own flexible and rigid videoendoscopes [5, 11].

Several channels make it possible to insert proper tools, manipulators, and other accessories, enabling to access the areas that were up to now inaccesible. External devices can aid these tools, e.g., electro-coagulation knives, lasers for cauterization, plasma argon coagulators. 
From the medical application point of view, the advantages of optical fiber systems include important known attributes such as great flexibility, low loss, small size, and noise avoidance. However, the applications of videoendoscopy used as the only imaging method are still limited. First, anatomic body structures make it possible to move fiberoscopes only inside natural tracts of the human organism. Furthermore, current endoscopic probes are not yet able to reach all parts of the small ducts. All units should meet the rigorous demands of endoscopic and other procedures to work comfortably and safely [4, 5].

The flat sight of endoscopic images causes operators to move their tools precisely inside the human body, relying on the existing monocular vision. Using a lot of manipulators allows getting new, virtually spatial experience. Such knowledge needs a lot of training to be mastered during a number of different interventions. Image sequences can be difficult to interpret anatomically. Computer methods and virtual endoscopy, which is based on the modelling of images to be obtained with computed tomography or magnetic resonance, can be helpful in training without real procedures [6].

Flat images with a significant distance to the operated areas and anatomical limitations require additional imaging techniques. Simultaneous use of X-rays in different planes allows, e.g., spatial visualization of the endoscopic device and other tool location in relation to operated organs (Fig. 1b). Insertion of stents inside various segments of alimentary tracts may be realized under continuous combined monitoring assisted by optical imaging with gastro-videoendoscopy. A stent is the endoprosthesis used to:

- hold tissue in place,

- keep an obstructed lumen patent,

- drain a tubular or ductal structure in cases of obstruction.

Stents are available in a variety of configurations featuring: different contours, diameters and lengths [11]. Alimentary stents are plastic, metal or silicone tubes designed to make open the obstructed area and help prevent it from becoming obstructed again. The main advantage of metal self-expanding stents compared to polymeric stents is the smaller diameter required for installation. Unexpanded stents are small enough to fit through the channel of an endoscope and they are cylindrical in shape.

The example of an esophageal stent is shown in Fig. 2. The goals of such stenting may be as follows:

- providing stents to bridge the obstruction caused by esophagus tumors,

- carrying out photodynamic therapy in the esophagus tumor area,

- providing stents to close fistulas,
- $\quad$ providing stents with anti-reflux valve (GERD: chronic GastroEsophageal Reflux Disease). a)

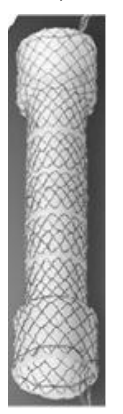

Fig. 2. View of an esophageal stent (a) and illustration of its placement (b), (c).
Examples of optical monitoring using videoendoscopy during successfully performed minimally invasive procedures are shown in Figs. 3, 4 and 5. The kinds of stenting technique included: 1) plastic catheter-like stents, 2) expandable metallic stents, 3) coupled application of stents and catheters with the so-called rendez-vous method. This method assisted by combined imaging may help to insert both metallic stents and plastic prostheses: the endoscope with a video-camera and a plastic prosthesis "meet" in the body site.

Optical imaging of successive implantation steps of a state-of-the-art self-expanding esophageal anti-reflux stent are presented in Fig. 3. Six successive videoendoscopic pictures have been selected from many images taken during the esophagus stenting procedure and its effect control.
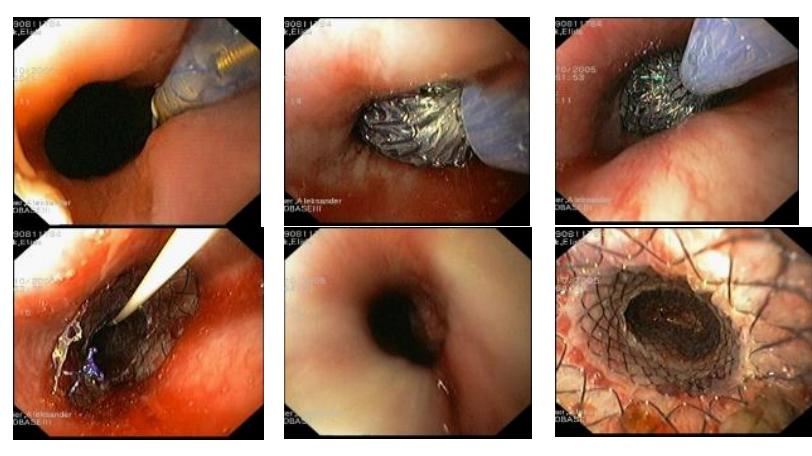

Fig. 3. Six succesive videoendoscopic images of esophagus stenting.

A nickel titanium stent was pre-mounted on an over-thewire delivery system: radioopaque markers are located at the ends of the stents. The markers identify the stent location. Imaging the application, we could notice how the stent provides a patent lumen. The stents, made of very thin wire mesh, were placed through the esophagus tumor using endoscopic guidance. The outer diameter of esophageal stent when unexpanded was ca. $4.5 \mathrm{~mm}$. When the stent was released it springs open. The success was 
observed after 24 hours in every procedure with receiving a complete diameter of stents. The examples of control Xray pictures of the implemented stent are shown in Fig. 4.
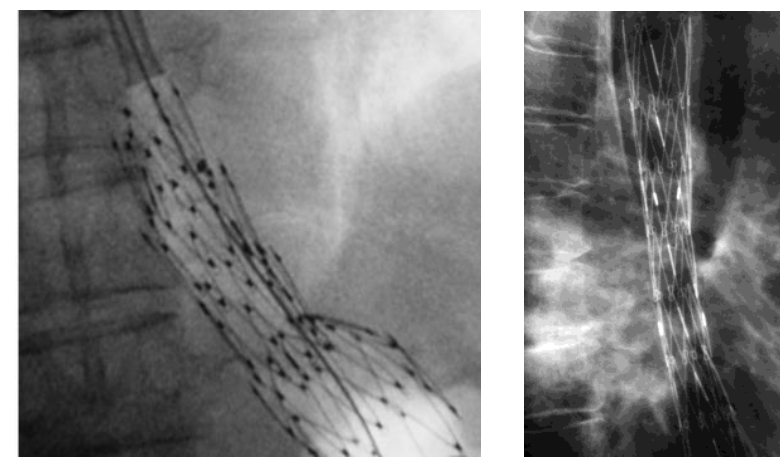

Fig. 4. Control X-ray pictures of the implemented esophagus stent

The stent was used for reinforcing weak walls and for widening narrow tubes (shape-memory materials are very elastic: when the deforming factor is released, the stent can return to its original shape).

A combined use of operation site imaging and the rendez-vous method also allowed to make numerous effective stenting and minimally invasive procedures. Making a hole in the wallstent net, the next self-expanding stent was implanted percutaneously. Two examples are shown in Fig. 5.
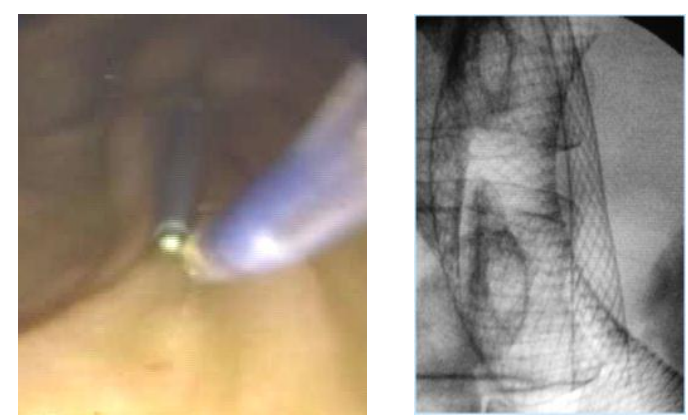

Fig. 5. Example of the rendez-vous procedure and combined imaging used to help insert metallic stents and plastic prostheses (left); view of two coupled self-expanding stents (right).

Particular methods of medical imaging are not alternative methods, but they can complement each other, according to the present tendencies in applying constantly developing combined imaging.

The interest in developing optical techniques in clinical practice to obtain images of tissues and organs is great and still growing. The role of optical techniques in current medical measurements and imaging is especially significant $[1,2]$. On the one hand, light scattering and absorption can complicate optical imaging [7, 8] and decrease image resolution in comparison with, e.g., the RTG techniques. On the other hand, it is possible to detect changes invisible on the X-ray pictures [1]. Thus, the complementary use of optical imaging may provide information on the functional condition unavailable in other techniques of medical imaging. Of course, progressive development of noninvasive optical imaging and measurements always depends very much on the clinical acceptance of advanced biomedical technology and engineering. Significant guidelines also refer to leaning, disinfection, and safety of endoscopes and stents application $[4,5,11]$. Biomedical engineers can play a significant part in this process.

In conclusion it may be stated that:

- Optical techniques used in videoendoscopy supported by X-ray scanning plus percutaneous procedures allow access to pathological objects located in places inaccessible so far. Sufficient results will only be obtained if the quality of images is sufficiently high, providing a reliable diagnosis;

- Efficient stenting depends on products which must meet various human and technical needs;

- Self-expandable metallic stents are significant advances made in modern palliative therapeutic gastro-videoendoscopy;

- Alimentary stenting can give hope to patients not qualified for classic therapy;

- Efficient medical stenting under technical control may be a good example of advanced clinical engineering giving hope to critically ill patients for improving the quality of their remaining life.

\section{References}

[1] A. Cysewska-Sobusiak, Phot. Lett. Poland 11, 2 (2019).

[2] P. Listewnik, A. Mazikowski, Phot. Lett. Poland 10, 3 (2018).

[3] J-S. Park, S. Jeong, and D. H. Lee, Clin. Endosc. 48, 3 (2015).

[4] B.S. Dhillon, Medical device reliability and associated areas (Boca Raton, CRC Press LLC 2000).

[5] J.F. Rey, R. Lambert, and The ESGE Quality Assurance Committee, Endoscopy 33 (2001).

[6] W.M. Saltzman, Biomedical engineering. Bridging medicine and technology (Cambridge University Press 2009).

[7] F.A. Duck, Physical properties of tissue: a comprehensive reference book (San Diego, Academia Press 1990).

[8] A. Cysewska-Sobusiak, Opt. Eng. 36, 4 (1997).

[9] A. Cysewska-Sobusiak, P. Skrzywanek, A. Sowier, IEEE Sensors Journal 6, 5 (2006)

[10] T. Mahmood, M.A. Scaffidi, R Khan, S.Ch. Grover, World Journal of Gastroenterol. 24, 48 (2018).

[11] https://medical.olympusamerica.com 